\title{
Acoustic mode confinement using coupled cavity structures in UHF unreleased MEMS resonators
}

\author{
Andreja Erbes ${ }^{1} \cdot$ Wentao Wang $^{2} \cdot$ Dana Weinstein $^{2} \cdot$ Ashwin A. Seshia $^{1}$ (D)
}

Received: 25 October 2017 / Accepted: 27 August 2018/Published online: 6 September 2018

(C) The Author(s) 2018

\begin{abstract}
This papers investigates device approaches towards the confinement of acoustic modes in unreleased UHF MEMS resonators. Acoustic mode confinement is achieved using specially designed mechanically coupled acoustic cavities known as acoustic Bragg Grating Coupler structures to spatially localize the vibration energy within the resonators and thereby improve the motional impedance $\left(R_{x}\right)$ and mechanical quality factor $(Q)$. This enhancement in the mechanical response is demonstrated with numerical simulations using distinct unreleased resonator technologies involving dielectric transduction mechanisms. These initial investigations show improvements in the $Q$ as well as enhanced vibrational amplitudes within the resonator domains (i.e. translating to improved $R_{x}$ values) in the case of coupled cavities as opposed to single cavity designs. An initial approach to fabricate the devices in a CMOS compatible dual-trench technology are presented.
\end{abstract}

\section{Introduction}

Modern RF and future mmWave communication devices rely on low phase noise oscillators and high performance band-pass transmission filters. The stringent requirements on the frequency selectivity for some of RF transceiver applications are traditionally achieved by Surface Acoustic Wave (SAW) resonators, bulk acoustic wave resonators (BAW) and/or partially released thin-film bulk acoustic wave resonators (FBAR).

For BAW and FBAR technology, the film thickness dictates the resonant frequency which therefore restricts devices operating at different frequencies being manufactured on the same die/wafer and monolithic integration. SAW technology is itself based on piezoelectric material substrates, incompatible with modern CMOS.

The demand for ultra-high frequency (UHF) and superhigh frequency (SHF) communication devices has generated interest in the development of alternative resonator technologies. There are still no mainstream foundry technology producing advanced-node CMOS technology with

Ashwin A. Seshia

aas41@cam.ac.uk

1 Department of Engineering, University of Cambridge, Cambridge, UK

2 EECS Department, Massachusetts Institute of Technology, Cambridge, USA high-performance released MEMS options on the same wafer. There is therefore a strong urge for monolithic integration of UHF and SHF band MEMS resonators/filters with complementary integrated circuits towards enabling small footprint, lower cost, high performance and low power wireless communication devices.

The recent development of MEMS resonators has made possible a direct implementation of micro-mechanical structures with electronic circuits. However, the majority of MEMS resonators still require a final release-step to create freely suspended vibrating structures (Wang and Weinstein 2011). This release step adds processing complexity to the manufacturing of monolithically integrated MEMS and CMOS. Furthermore, to achieve high mechanical $Q$ and to effectively confine the mechanical vibration solely within the resonators, vacuum conditions are required to minimize the damping due to air and other viscous effects, which increases the packaging constraints and complexity.

To overcome the need of post-processing release steps, the technology described in Wang and Weinstein (2012a) using deep-trench capacitors (DT) has enabled a new route towards the integration of MEMS resonators and the CMOS. This paper builds upon this technology as well as replicated phonic-crystal (PnC) structures to propose a device methodology comprising multiple coupled acoustic cavities for acoustic mode confinement in order to achieve high $Q$ factors. 


\section{Models}

\subsection{Matrix formulation}

In order to model and design unreleased resonators, multicavity coupled structures, classical waveguide/transmission line theory is used to develop simple numerical simulation algorithms of the one-dimensional models. At the UHFSHF bands of operation, the wavelengths of acoustic waves traveling in CMOS compatible materials (e.g. Si, poly-Si, $\mathrm{SiO}_{2}, \mathrm{Al}$, etc.) are in the order of microns and therefore optical lithography can be used to tune the device operating frequency.

Figure 1 shows the unit-cell in a multi-layered periodic structure using two distinct acoustic materials with impedance $Z_{1}$ and $Z_{2}$.

In each medium, assuming no dispersive effects, the wave vector $k$ is a function of frequency $\omega$ and acoustic longitudinal wave speed $v_{L}$ through the linear relation

$k=\frac{2 \pi}{\lambda}=\frac{\omega}{v_{L}}$.

The effective acoustic impedance $Z$ can be defined using (1) as in Auld (1990)

$Z=\frac{c_{11}}{v_{L}}=\sqrt{\rho c_{11}}=\sqrt{\frac{\rho E(1-v)}{(1+v)(1-2 v)}}=\rho v_{L}$.

In the special case of a single interface between Medium 1/ Medium 2, assuming incoming waves from Medium 1, the transmission $T_{1 \rightarrow 2}$ and reflection $R_{1 \rightarrow 2}$ coefficients are

$T_{1 \rightarrow 2}=\frac{a_{2}}{a_{1}}=\frac{2 Z_{1}}{Z_{1}+Z_{2}}, \quad R_{1 \rightarrow 2}=\frac{b_{1}}{a_{1}}=\frac{Z_{1}-Z_{2}}{Z_{1}+Z_{2}}$.

From (3), the reflection and transmission parameters are adjusted by employing different CMOS compatible material pairs (e.g. $\mathrm{Si} / \mathrm{SiO}_{2}, \mathrm{Si} /$ poly-Si, etc.). Large and low acoustic impedance index materials are defined with letters

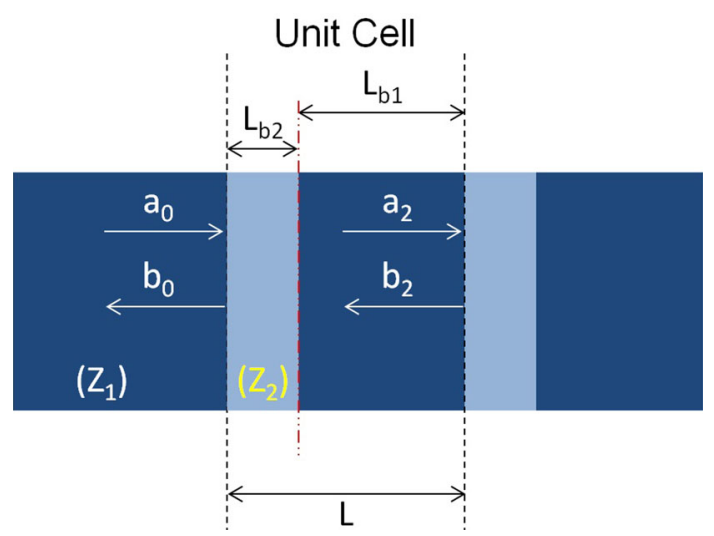

Fig. 1 Diagram of a 1D unit-cell of a multi-layered periodic structure using two distinct acoustic materials with impedance $Z_{1}$ and $Z_{2}$
$H$ and $L$, respectively, where the $H / L$ sections can have arbitrary lengths.

\subsection{Acoustic Bragg reflectors (ABR)}

The ABR (Wang and Weinstein 2011) uses the existence of propagation bandgaps in a periodic replication of a $H / L$ index to produce a reflective structure. The spatial periodicity is chosen such that the length of the unit cell is close to $\frac{\lambda_{0}}{2}$ where $\lambda_{0}$ is the required central wavelength of the mirror. Such Bragg reflectors are commonly used in solid mounted resonators, where the ABR multilayer structures are located vertically below the main acoustic cavity.

By reflecting back the waves dissipated in the bulk of the material, the $Q$ of the cavity can be increased as the dissipated energy per unit cycle is reduced

$Q=2 \pi \frac{E_{\text {stored }}}{E_{\text {Lost-per-cycle }}}$.

The energy lost in one cycle is directly proportional to the reflectivity of the mirrors surrounding the resonant cavity. Recently, such ABRs have been employed to produce unreleased MEMS resonators (Wang and Weinstein 2012b).

The contour plots of the $\left|S_{11}\right|$ parameter can be simulated for different ABR sizes and normalized frequency, as shown in Fig. 2. The lengths of the $L_{b 1}$ and $L_{b 2}$ sections are chosen to be $\lambda / 4$ length at the design frequency. From Fig. 2, as the ABR size is increased, a frequency band-gap is formed in which incoming waves are fully reflected, with an approximate frequency bandwidth of $300 \mathrm{MHz}$ at a central frequency of $3 \mathrm{GHz}$. The existence of these

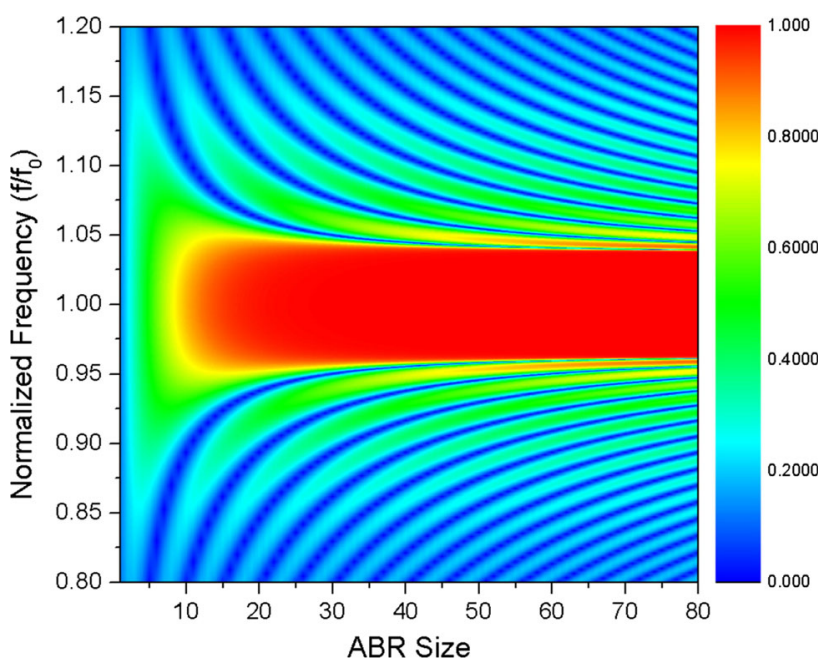

Fig. 2 Contour plot of the reflection coefficient $\left|S_{11}\right|$ for different $\mathrm{ABR}$ sizes and frequency range (normalized frequency $f_{0}=3 \mathrm{GHz}$ ) 
frequency band-gaps is fundamental to the acoustic confinement of vibration modes in a cavity surrounded by ABRs.

\subsection{Dispersion relation and band diagrams}

To simulate the band diagrams in the case of one-dimensional reflectors, ABR structure are assumed to extend to an infinite number of $H / L$ periodic repetitions of the unit Bragg cell. Floquets Theorem, which states that the spatial periodicity of the geometrical structure will be translated in a spatial periodicity of the wave-equation solution, can be applied to the unit-cells.

Defining $D=L_{B 1}+L_{B 2}$ as the spatial periodicity of the ABR structure and applying Floquet's theorem (Tamura et al. 1988), the following dispersion relation between the wave vector $q$ and the frequency $\omega$ can be found

$$
\begin{aligned}
\cos (q D)= & \cos \left(\frac{\omega L_{B 1}}{v_{1}}\right) \cos \left(\frac{\omega L_{B 2}}{v_{2}}\right) \\
& -\frac{1+\delta^{2}}{2 \delta} \sin \left(\frac{\omega L_{B 1}}{v_{1}}\right) \sin \left(\frac{\omega L_{B 2}}{v_{2}}\right)
\end{aligned}
$$

where $\delta=\frac{Z_{1}}{Z_{2}}$ is the ratio of the acoustic impedances.

\subsection{Engineering the width of the frequency band-gap}

The width of the band-gap can be engineered to achieve a particular central frequency through an appropriate choice of material-pair and Bragg-unit cell pitch size. Within that frequency gap, there is no real-valued solution of the wave equation and therefore, no acoustic mode is able to propagate throughout the structure (as they would in a waveguide). This leaves place only for evanescent waves which have a spatial decay length of $\alpha_{D}$ (Joannopoulos et al. 2008). The irreducible Brillouin zone for this $1 \mathrm{D}$ periodic structure is $-\pi / D<q<\pi / D$. This section considers the specific case of isotropic Silicon $(\mathrm{Si}$ (iso)) and $\mathrm{p}$-Si with acoustic impedance $Z_{1}$ and $Z_{2}$, respectively, as the materials forming the Bragg unit cell.

The dispersion plots for different unit Bragg-cell configurations (i.e. different $\alpha_{i}$ coefficients) can be evaluated, as shown in Fig. 3. This is done by solving (5) for different unit Bragg-cell designs:

(1) $\mathrm{Si} L_{B 1}=\frac{\lambda_{1}}{4}$ and $\mathrm{p}-\mathrm{Si} L_{B 2}=\frac{\lambda_{2}}{4}$,

(2) Si $L_{B 1}=0.3 \lambda_{1}$ and p-Si $L_{B 2}=0.2 \lambda_{2}$,

(3) Si $L_{B 1}=\frac{\lambda_{1}}{3}$ and p-Si $L_{B 2}=\frac{\lambda_{2}}{6}$,

(4) Si $L_{B 1}=0.4 \lambda_{1}$ and p-Si $L_{B 2}=0.1 \lambda_{2}$.

For all four cases, the pitch-size is defined as $D=L_{B 1}+L_{B 2}$, which is also the dimension of the unitcell. From Fig. 3, as the designs deviate from the quarter-

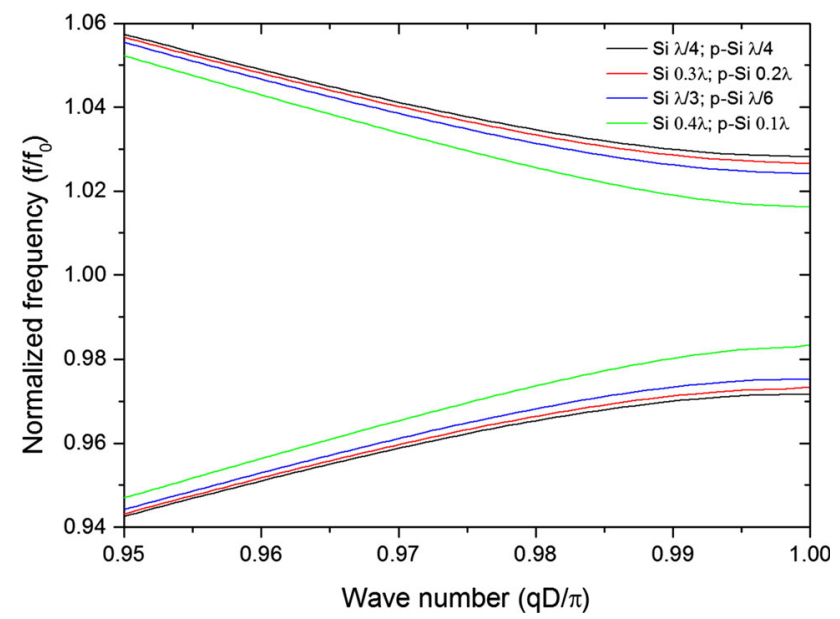

Fig. 3 Simulated dispersion relation for normally incident waves to an infinite ABR structure of poly-silicon/silicon for different unit Bragg-cell designs

wavelength/quarter-wavelength structure of design 1 , the width of the band-gap decreases. This has a direct impact on the number of reflectors $N$ required in an ABR to achieve a near $100 \%$ reflection coefficient, as it was already seen from the different cases plotted in Fig. 2. A diminished band-gap as in design 4 also implies a non-zero phase shift between the incident wave and the fully reflected wave from the ABR. The width of the band-gap is also dependent on the acoustic mismatch $\delta$ of the materials chosen.

The design and optimization of a single acoustic cavity embedded in a substrate material is described in Wang and Weinstein (2012b). By using ABRs, the $Q$ and $R_{x}$ of unreleased MEMS resonators can be improved as shown in Wang and Weinstein (2012a, b).

\subsection{FEM computation of dispersion relations}

While the one-dimensional results derived previously only considered propagation of longitudinal waves with wave vectors collinear to the traveling direction, manufactured devices have much more complexity that is best captured in a numerical model. The complexity in the geometry as well as solid elastic wave nature of the materials used will generate an acoustic coupling of longitudinal and shear waves which interact differently with the unit-cells and allows for many other guided modes to propagate within such periodically replicated structures. Dispersion plots are generated in order to identify all the possible acoustic modes such structures can couple energy into.

Figure 4 shows a simulated plot of the two principal longitudinal mode shapes for the particular ABR design. Mode 1 is found at $2.652 \mathrm{GHz}$ while Mode 2 is located at $2.684 \mathrm{GHz}$, which gives an effective frequency band-gap 


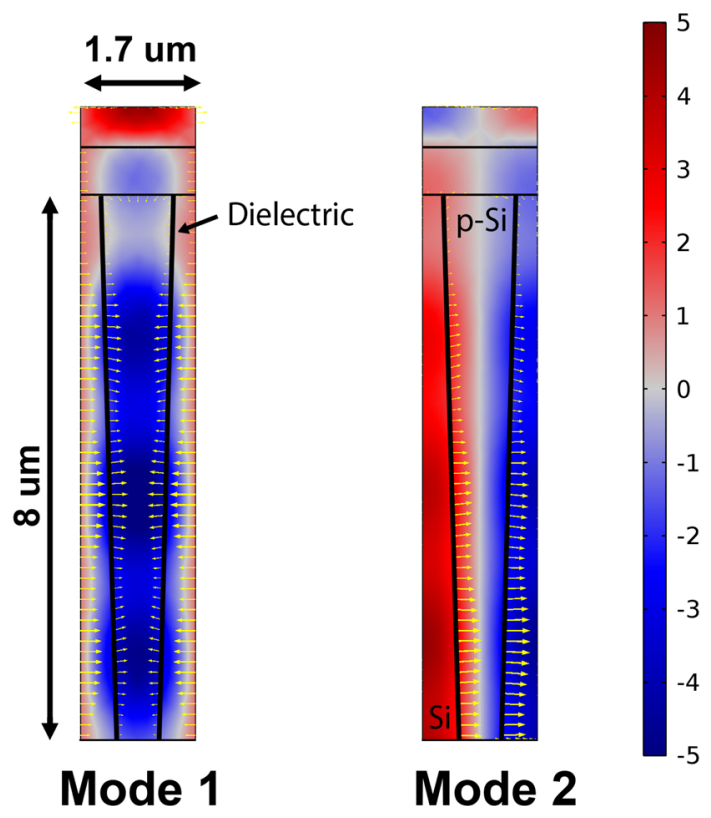

Fig. 4 Simulated principal longitudinal mode shapes assuming a Floquet periodic ABR structure. The contour map represents the longitudinal strain distribution and the arrows represent the local material displacement

of $32 \mathrm{MHz}$-which is $5.3 \times$ smaller than what was simulated in the $1 \mathrm{D}$ case on equivalent $3 \mathrm{GHz}$ ABRs. Furthermore, a more precise study of the mode shapes shows strong transverse displacements (e.g. in Mode 1) which extend infinitely in the bulk of the material. Since the ABRs are buried in a relatively thick substrate, a large number of transverse modes with lower/higher frequencies are allowed to fully propagate in the transverse direction, without any $\mathrm{ABR} /$ phononic structures preventing them from leaking vibration energy out of the cavities and hence, degrading the $Q$.

Figure 5 shows the simulated dispersion curves using COMSOL for the ABR structure presented in Fig. 4.

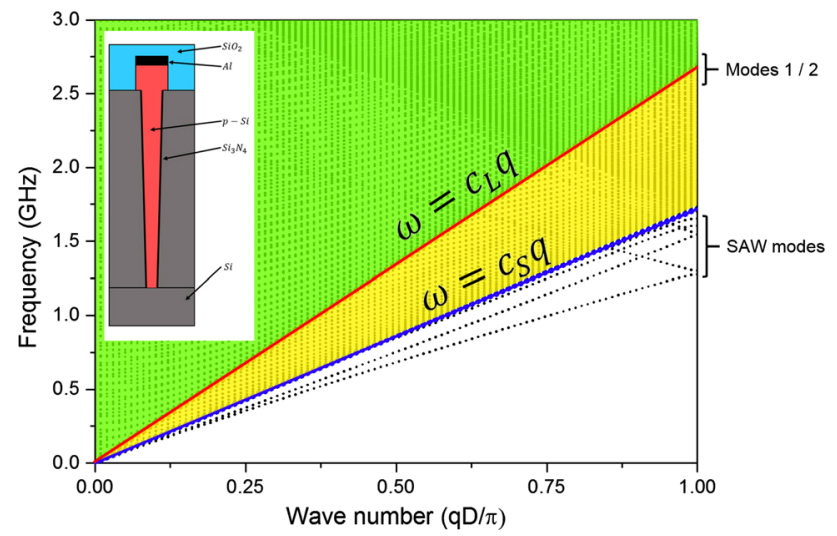

Fig. 5 Simulated dispersion curves using COMSOL for the ABR structure of Fig. 4 with longitudinal sound cone (red curve) and shearwaves sound cone (blue curve) (colour figure online)
Included in these plots are also the two sound lines or sound cones (Joannopoulos et al. 2008) which represent the $\omega=c q$ lines for the longitudinal (red curve) and shearwaves (blue curve) regions. The black dots represent accessible modes of vibrations which would exist in an infinitely-repeated structure. From Fig. 5, below the sound cone of the shear waves lies only a finite number of branches or available modes of vibrations. These modes are well separated in the frequency domain and because of their location below the shear-wave sound cone, they are fully guided within the ABR structure which acts as an acoustic wave-guide (Bahr et al. 2015). For a given wavenumber $q$, there is a threshold frequency at which there is a near-continuum space of available modes which are able to travel freely within the structure. This availability of spurious modes, which are not accessible in purely one-dimensional simulations, are one of the possible reasons explaining the degradation of the $Q$ of manufactured longitudinal-mode resonators using these ABRs.

Furthermore, in a two-dimensional structure lying in the $(x, y)$ plane, any traveling wave vector $\mathbf{k}$ will have an orthogonal decomposition as

$\mathbf{k}=k_{x} \underline{e_{x}}+k_{y} \underline{e_{y}}$

where $e_{x}$ and $e_{y}$ are the unit base vectors in the $x$ and $y$ directions, respectively.

While the longitudinal component $k_{x}$ of the $q$ wavevector are only able to trigger the modes shown in the dispersion diagrams (i.e. satisfying stringent Floquet equations), any additional transverse components $k_{y}$ of $q$ are able to travel freely as they are not restricted by any periodic structure in the transverse direction (as long as the frequencies are located above the sound cone lines).

\subsection{Modal coupling and impact on the mechanical $Q$}

The unreleased coupled resonator structures are to be operated in bulk longitudinal vibration modes at frequencies above $1.5 \mathrm{GHz}$. From Fig. 5, there is no perfect bandgap when $q=\pi / D$, which is precisely where the two main longitudinal modes of the reflectors of Fig. 4 are located. In this region, acoustic modes lie well above the shear sound cone for which there exists a near continuum of solutions to the wave equations; these wave vectors $q$ are guided into the $x$-direction and can travel freely in the $y$-direction. This mechanism of modal coupling to higher order longitudinal/ shear modes is one of the main reasons why such unreleased resonators have finite $Q$ s. It also explains why the vibration energy does not stay confined within the resonant cavities and eventually radiates out into the substrate. Resonant cavities which are solely surrounded by such 
ABR structures will always have a loss mechanism associated with the presence of a near-continuum of eigenmodes in which the vibration energy can couple into.

One way to reduce the modal density at these frequencies is to reduce the thickness of the substrate (i.e. towards a fully released structure). Alternatively, to improve the performance of unreleased resonators, the targeted resonant modes need to be designed in such a way that they remain within the longitudinal band-gap formed by the ABR, while at the same time minimizing the coupling to other spurious modes. One way to reduce modal coupling is to have a slight frequency offset between the eigenfrequencies of the cavity resonant modes and the $m$ th order center frequency $\omega_{m}$ of the mirror's band-gap. This was shown in Joannopoulos et al. (2008) to reduce the modal coupling and radiation to the surrounding materials which is largest at $\omega_{m}$.

However, operating the resonators away from $\omega_{m}$ allows a fraction of the energy to escape in the longitudinal direction through the reflectors. This can be explained by the duality between the spatial domain and the frequency space: any vibration distribution in the cavity (i.e. distribution in the $x$-space and time $t$ ) will have a unique frequency distribution (i.e. in the $k$ and $\omega$ space).

The $(k, \omega)$ representation of the targeted vibration modes $(x, t)$ can be mapped onto the dispersion plots and overlaid on those of the ABR. Therefore, a strongly localized mode in the $(x, t)$-space will have a near-uniform distribution in the $(k, \omega)$ space. This uniform distribution allows for some $q$ values to coincide with eigenmodes of the ABR which inherently leak vibration energy out of the cavity and degrade the resulting $Q$ and $R_{x}$ of the resonator. On the other hand, having a unit delta distribution in the $(k, \omega)$ space in which there is no spurious modal coupling leads to a uniform $(x, t)$ distribution, which means that the mode would have to extend indefinitely from the cavity into the surrounding ABR. There is therefore a trade-off between the amount of spatial localization within the cavity (i.e. improved $R_{x}$ ) which will result in energy being coupled to many other surrounding modes (i.e. degraded $Q$ ), and, the delocalization to reduce this effect but increase the amount of energy leakage through the surrounding ABRs (i.e. degraded $R_{x}$ ).

One potential route towards the improvement in performance of such unreleased resonators is to investigate the impact of mechanical coupling of multi-cavity resonators and the vibration localization resulting from such coupled systems on both the $R_{x}$ and $Q$ measures. As found in Joannopoulos et al. (2008), a gradual variation in the structural properties within a photonic/phononic crystal slab can significantly improve the $Q$ of the resonators. Furthermore, other methods exist where it is possible to retain the benefits of a localized mode by operating right in the center of the band-gap, while still recording an increase in the $Q$. This was reported in Johnson et al. (2001) where the multipole-radiation patterns are canceled through an appropriate design of the cavities. Mechanically coupled unreleased cavities comprising defined structural perturbations can essentially mimic this gradual structural change (i.e. going from the bulk substrate, the surrounding mirrors and towards central cavities).

\section{Mechanically coupled unreleased resonators}

In order to improve the $Q$ and $R_{x}$ of unreleased resonators without the requirement for an excessively large number of ABRs, we propose an architecture comprising of acoustically coupled unreleased multi-cavity resonators. The benefit of using mechanically coupled resonators is to leverage phenomena such as the localization of the acoustic energy (e.g. as described in Thiruvenkatanathan et al. 2011). For instance, in the case of unreleased resonators, the acoustic localization of the mechanical vibration energy can be used to confine the vibration energy only in some regions of interest (i.e. the resonators: improving the $R_{x}$ ) and minimizing the loss of energy to the surroundings and the substrate (i.e. enhancing the $Q$ ).

From Fig. 5, there is a large modal density in the region of interest as there is a near continuum of allowable states in the vicinity of the longitudinal sound line (i.e. region with significant modal-overlap). Multi-cavity designs have therefore the potential to improve the distribution in the $(k, \omega)$ space and thereby reduce the activation of adjacent modes and ultimately, improve the $Q$. In order to investigate the effect of mode confinement and energy localization using coupled unreleased cavities and implement novel methods for acoustic confinement, efficient acoustic coupling structures are proposed.

\subsection{Acoustic Bragg grating coupler (ABGC)}

The ABGC is defined as a specially designed multilayerstructure of an $N$-fold periodic replication of a high/low index layer structure $(H L)^{N}$ which allows for the transmission of vibration energy and improves the reproducibility of the achieved coupling strength of unreleased acoustic cavities.

Figure 6 is a SEM image of a micro-fabricated 2 cavity coupled structure in the case of $H$ : Silicon/ $L$ : poly-silicon materials, where different structural designs are used for the $\mathrm{ABGC}$ section and the ABR reflectors.

The parameters of the ABGC design will modify the coupling strength between Cavity 1/Cavity 2 and modify 


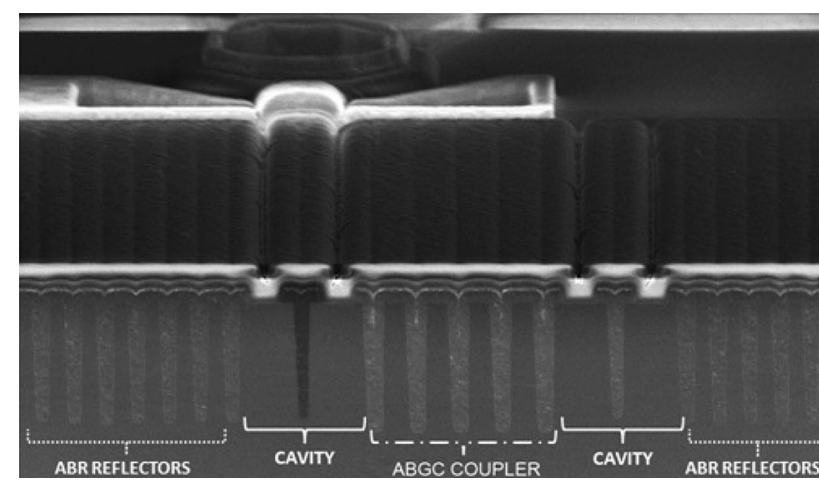

Fig. 6 SEM of a micro-fabricated mechanically coupled unreleased acoustic cavities resonator

the allowable eigenmodes. The ABR unit-cell and geometry can be fully optimized using the dispersion relations covered in the previous sections. The main aim is to achieve the largest band-gap and reduce the coupling of longitudinal modes to the transverse components and other spurious modes. Likewise, the unit-cell of the ABGC can be fully optimized using the two-dimensional dispersion curves.

Throughout the next subsections, families of coupled resonator designs will be defined as

$$
\begin{gathered}
\operatorname{ABR}\left(N_{1}\right)|\operatorname{CAVITY}(1)| \ldots \operatorname{CAVITY}(j) \ldots \\
\ldots A B G C\left(N_{j}\right)|\ldots C A \operatorname{CITY}(N)| \operatorname{ABR}\left(N_{1}\right),
\end{gathered}
$$

where $A B R\left(N_{i}\right)$ and $A B G C\left(N_{j}\right)$ defines $\mathrm{ABR}$ and ABGC structures with replication periods of $N_{i}$ and $N_{j}$, respectively, and $\operatorname{CAVITY}(j)$ is the $j$ th type of cavity design.

\subsection{Optimizing cavity lengths}

In order to study the effect of the ABGC couplers, ABR reflectors and number of cavities on such manufactured resonators, the matrix formalism introduced in the previous sections is used to simulate the amplitude transmission response by computing the $S_{21}$ transmission coefficient as a function of the normalized input frequency (e.g. in this case $f_{0}=2.74 \mathrm{GHz}$ ).

This section presents optimization procedures for multicavity structures, starting with the selection of cavity lengths.

\subsubsection{Single cavity}

For a given set of reflectors, coupler number and cavity number, there are optimum values of cavity lengths which yield to eigenmodes where the largest amplitude of vibration occurs in the vicinity of the central cavities. In multicavity configurations, many peaks are induced due to coupling and it becomes crucial to be able to track the different eigenfrequencies and eigenmodes as a function of the chosen cavity designs. This can be done with numerically simulated contour plots of transmission coefficients through the structure as a function of both the cavity length and frequency.

Figure 7 shows the contour plot of the transmission coefficient through an unreleased, single cavity resonator as a function of normalized drive frequency $\left(f_{0}=3 \mathrm{GHz}\right)$ and cavity length. For this resonator, the cavity is surrounded by $50 \mathrm{ABR}$ reflectors where the unit Bragg-cell section is taken as quarter-wavelength elements at a center frequency of $f_{0}=3 \mathrm{GHz}$. From Fig. 7, low transmission regions are identified as the frequency band-gaps due to the outer ABR reflectors. However, the unreleased cavity allows for very narrowband transmission peaks within that band-gap for specific frequency-cavity length combinations.

With these two-dimensional plots, it becomes easier to locate the optimum cavity design lengths. At $3 \mathrm{GHz}$, a $\lambda / 2$ section in $\mathrm{Si}\langle 110\rangle$ direction has a length of $1.4 \mu \mathrm{m}$. This means that odd multiples of $\lambda / 2$ are found for cavity lengths of $1.4 \mu \mathrm{m}, 4.2 \mu \mathrm{m}$ and $7 \mu \mathrm{m}$.

When operated at the center of the frequency band-gap, the ABRs will fully reflect with a near-zero phase lag, allowing for odd multiples of half-wavelengths standing waves (i.e. acoustic modes with a zero displacement in the center of the cavity) to grow. Similarly, even-multiples of half-wavelength transmission peaks are also allowed within these cavities. Evaluating the mode shape at a given eigenfrequency is crucial when designing the electro-mechanical transducers for $R_{x}$ reduction.

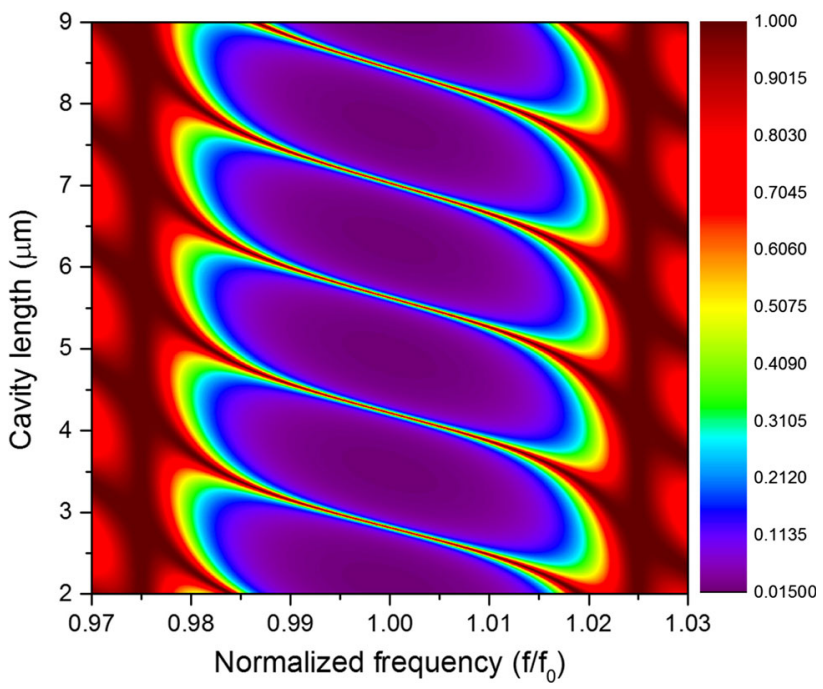

Fig. 7 Contour plot of the transmission coefficient of a single cavity resonator for different cavity lengths and normalized drive frequen$\operatorname{cies}\left(f_{0}=3 \mathrm{GHz}\right)$ with $50 \mathrm{ABR}$ reflectors $\left(L_{b 1}=L_{b 2}=\lambda / 4\right.$ at $\left.3 \mathrm{GHz}\right)$ 


\subsubsection{Five coupled cavities}

Figure 8 shows the contour plots of the transmission coefficient through an unreleased, five coupled cavities resonator as a function of the normalized drive frequency $\left(f_{0}=3 \mathrm{GHz}\right)$ and cavity length. The coupling is chosen such that $\operatorname{ABGC}\left(N_{2}\right)=\operatorname{ABGC}\left(N_{1}\right)=3$. From Fig. 8, there are now many more transmission peaks within the two-dimensional contour plots corresponding to the presence of multiple acoustic modes within the five coupled cavities.

It also shows the complexity of the design space as many different parameters can be modified and will result in a variety of responses. For example, one could study the effect of having different unit-cells designs for the ABR and the ABGC. Furthermore, in order to enhance further the vibration localization within the central cavities, one could design non-symmetric cavity designs where the outer cavities would have different lengths as compared to the central cavities.

The benefits of having coupled resonators for the enhancement of spatial localization of the vibration mode can be demonstrated by studying the response of a five coupled cavity resonator and comparing it with a single cavity resonator. In both cases, the Bragg-unit cell for the ABR and the ABGC is chosen such that $L_{B 1}=L_{B 2}=0.75 \mu \mathrm{m}$ in a $\mathrm{Si} / \mathrm{p}-\mathrm{Si}$ configuration. For the five coupled cavities, $\operatorname{ABGC}\left(N_{2}\right)=4, \operatorname{ABGC}\left(N_{1}\right)=8$ and both structures are surrounded by 50 ABR pairs. After producing two-dimensional contour plots as those found in Figs. 7 and 8, the optimal cavity lengths are found as $L_{1 C A V}=4.54 \mu \mathrm{m}$ and $L_{5 C A V}=4.04 \mu \mathrm{m}$ for the single cavity and five coupled cavities resonators, respectively.

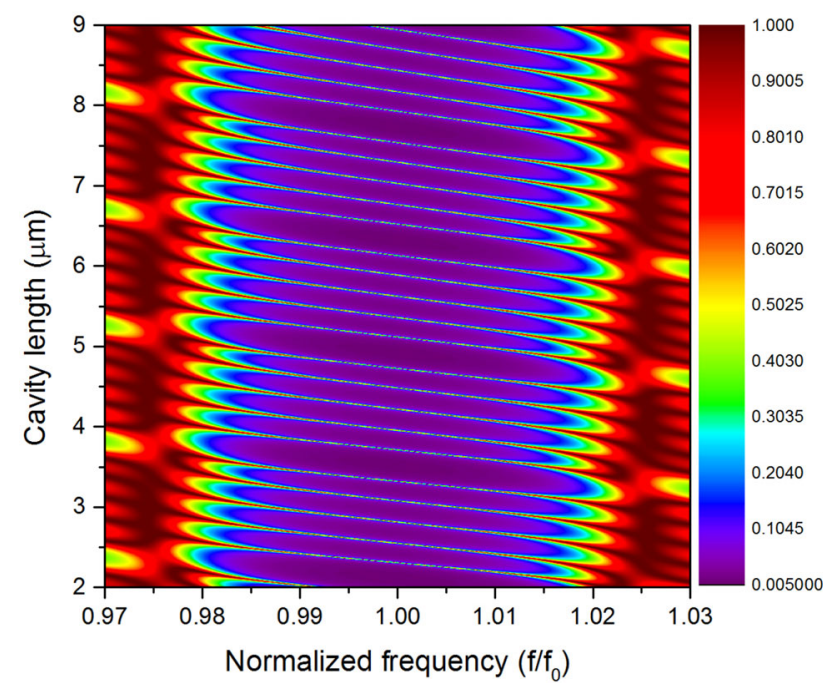

Fig. 8 Contour plot of the transmission coefficient of a five coupled cavities resonator for different cavity lengths and normalized drive frequencies $\left(f_{0}=3 \mathrm{GHz}\right)$ with $50 \mathrm{ABR}$ reflectors $\left(L_{b 1}=L_{b 2}=\lambda / 4\right.$ at $3 \mathrm{GHz}), \operatorname{ABCG}\left(N_{2}\right)=\operatorname{ABGC}\left(N_{1}\right)=3\left(L_{b 1}=L_{b 2}=\lambda / 4\right.$ at $\left.3 \mathrm{GHz}\right)$
Figure 9 shows the simulated eigenmode of the two structures corresponding to the transmission peaks located at $f / f_{0}=1$. From Fig. 9 , the benefits of employing multicavity structures can been see in terms of the achieved enhancement of the displacement amplitude levels as opposed to the single cavity resonator. These simple onedimensional simulations can be readily used to quickly determine initial designs for multi-cavity structures for the improvement of the $R_{x}$.

\section{3 $R_{X}$ and $Q$ optimization}

Just as in the case of weakly coupled released resonators, perturbations induced in the structurally symmetry can significantly affect the vibration dynamics and energy distribution within the system (Erbes et al. 2015).

In the case of unreleased resonators, this can be done by using asymmetric resonator designs (e.g. having multicavity structures with ABGC sections of different strengths), or by inducing perturbation and/or defects within the structures (e.g. by having cavities of different sizes within a coupled structure). Other methods include the gradual change in the structural designs of the different building blocks of the coupled resonators. To evaluate the potential of cavity perturbation on resonator metrics, consider the example of five coupled cavities with the following structure

$$
\begin{aligned}
& A B R(50)\left|C A V 1\left(L_{0}\right)\right| A B G C\left(N_{1}=4\right)\left|C A V 2\left(L_{02}\right)\right| \\
& A B G C\left(N_{2}=8\right)|| C A V 3\left(L_{01}\right)\left|A B G C\left(N_{2}=8\right)\right| \\
& C A V 4\left(L_{02}\right)\left|A B G C\left(N_{1}=4\right)\right| C A V 5\left(L_{0}\right) .
\end{aligned}
$$

We can define the dimension $L_{0}$ is defined as the length of Cavities 1 and 5, $L_{01}=p_{1} L_{0}$ as the length of Cavity 3 and

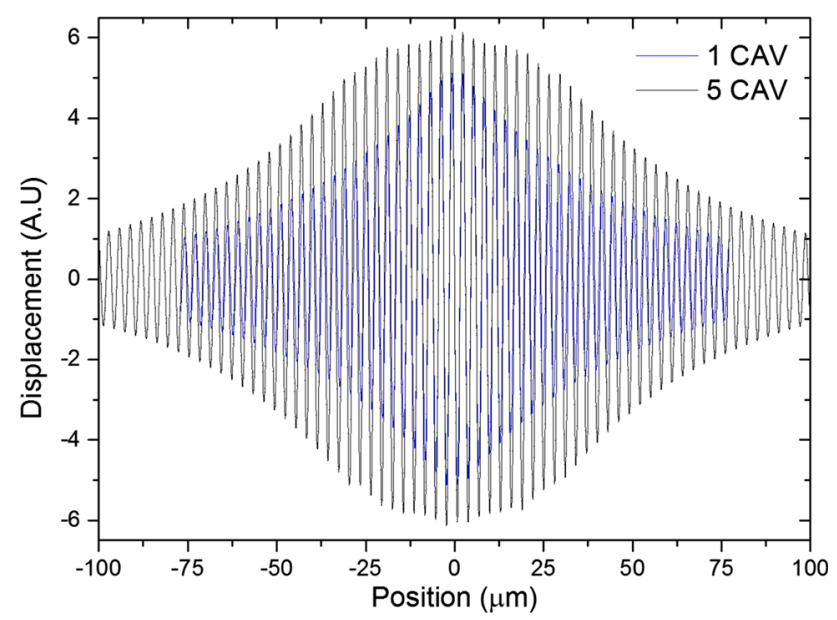

Fig. 9 Simulated vibratory mode shape for the single cavity (1 CAV blue curve) and five cavity (5 CAV black curve) resonators (colour figure online) 
$L_{02}=p_{2} L_{0}$ as the perturbed length of Cavities 2 and 4 . In order to achieve the best performance from the ABR mirrors, a structure is considered as optimized when the particular mode of interest is located right at the center of the band-gap. This is done by using the two-dimensional contour plots presented in earlier sections. Three distinct cases are evaluated for this study:

(1) Case 1: $L_{0}=3.56 \mu \mathrm{m}, p_{1}=p_{2}=0, f l f_{0}=1$,

(2) Case 2: $L_{0}=3.73 \mu \mathrm{m}, p_{1}=0.14, p_{2}=0.1, f / f_{0}=1$,

(3) Case 3: $L_{0}=3.87 \mu \mathrm{m}, p_{1}=0.14, p_{2}=0, f l f_{0}=1$.

In all three cases the particular mode of interest is located at the center of the band-gap (i.e. $f / f_{0}=1$ ).

Figure 10 shows the simulated vibratory mode shapes for the three cases. Case 3, which features a perturbation of $14 \%$ in the central cavity, is able to achieve the largest localization of the vibration amplitude in the center of the resonator. Case 1, on the other hand, has a symmetric structure which translates in having a mode which extends much more uniformly throughout the five cavities. Compared to the single cavity design of Fig. 9, all three cases show enhanced localization of the vibration energy and achieve larger amplitudes of vibration thus enabling smaller $R_{x}$ for these structures.

The presence of these small perturbations in the cavity lengths affects the density of available modes within the ABR band-gap. Figure 11 are simulated contour plots of the transmission coefficient for Case 1 (Fig. 11a) and Case 3 (Fig. 11b), respectively. The eigenmode of interest (i.e. the one plotted in Fig. 10) is marked with the white dashed circle on both contour plots.

From Fig. 11a, Case 1, which achieves a broader spatial vibration distribution compared to the other two cases, is located at $f / f_{0}=1$. However, at the particular cavity length $L_{0}=3.56 \mu \mathrm{m}$, there is another eigenmode in its

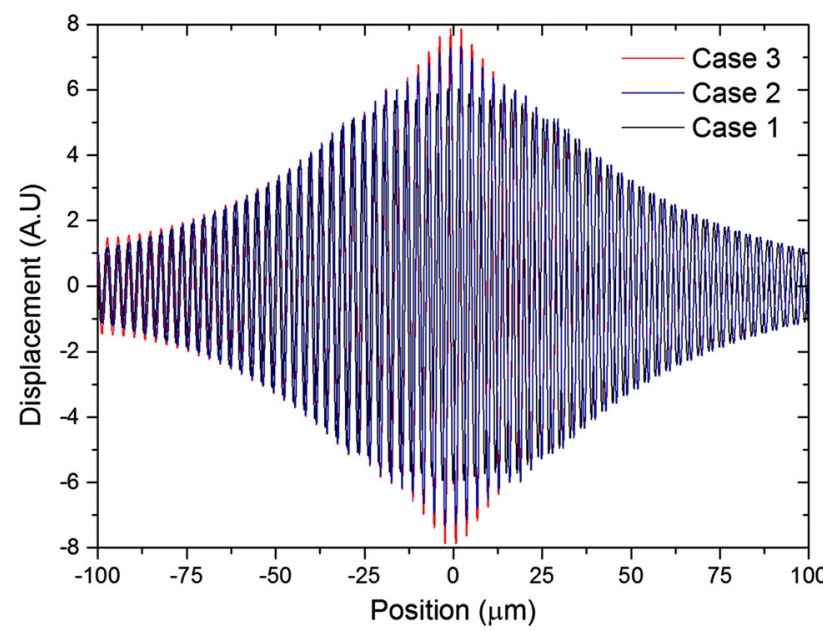

Fig. 10 Simulated vibratory mode shape for the single cavity a five cavity resonator with different perturbations
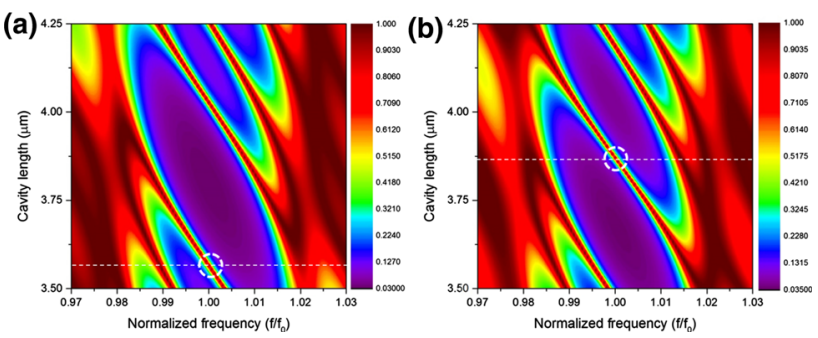

Fig. 11 Simulated contour plots for the resonator structures of a Case 1 , b Case 3

vicinity (at $f / f_{0}=0.99$ ). This is not the case in Fig. $11 \mathrm{~b}$ of Case 3 which is nearly isolated from the surrounding modes for a given horizontal slice at $L_{0}=3.87 \mu \mathrm{m}$. The perturbations induced through the coefficients $p_{1}$ and $p_{2}$ are used to isolate a particular mode from the others and therefore optimize the localization of the eigenmode.

In order to evaluate the benefit of mode confinement on the $Q$ of these resonators, FEM analysis are performed in order to simulate the full interaction between the resonant cavities, the ABRs and the bulk of the material.

\subsection{Cavity length scanning}

To examine the effects of manufacturing variations, simulations of the structures are conducted as a function of varying cavity length to examine the impact on the response of the cavity modes. Figure $12 \mathrm{a}$ shows the

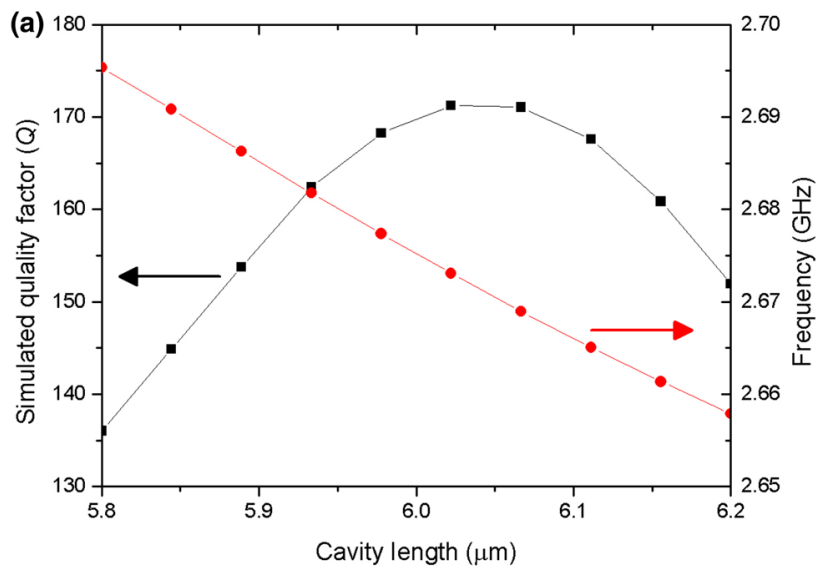

(b)

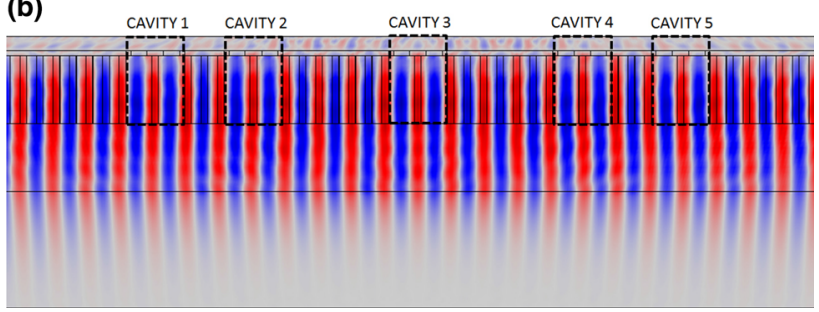

Fig. 12 a Simulated quality factor $Q$ and resonant frequency, for different cavity lengths. b Simulated strain field for the largest $Q$ case when $L_{\text {cav }}=6.02 \mu \mathrm{m}$ for a five coupled cavities resonator 
simulated quality factor $Q$ and resonant frequency, for different cavity lengths and Fig. 12b the simulated strain field for the largest $Q$ case when $L_{\text {cav }}=6.02 \mu \mathrm{m}$ for a five coupled cavities resonator. Similar dependence between the transmission coefficient and the cavity length was found in two-dimensional contour plots as in Fig. 11. It can be seen from Fig. 12a that there is an optimal cavity length for which the $Q$ is maximized (i.e. $Q \approx 170$ for $\left.L_{\text {cavity }} \approx 6.05 \mu \mathrm{m}\right)$.

From Fig. 12b, for such resonator designs, the vibration extends deeply into the substrate. The reflectance of the $\mathrm{Si} /$ poly-Si Bragg-sections is just not large enough to confine the vibration in the resonator slab. However, having $\mathrm{ABR}=50$, the five cavity design (which has an overall resonator length $L_{\mathrm{res}}=240 \mu \mathrm{m}$ ) is able to achieve similar $Q$ values as those for much longer single cavity designs with $\mathrm{ABR}=120\left(L_{\mathrm{res}}=420 \mu \mathrm{m}\right)$. Furthermore, the five cavity resonators has an improved signal output compared to the single cavity resonator as demonstrated in Fig. 9.

\section{Electromechanical transduction}

The general driving mechanisms of unreleased structures are analogous to piezoelectrically driven resonators and those featuring an internal dielectric transduction. In both cases, the transducer element is located at the point of maximum strain. The design and analysis of SHF silicon bulk mode resonators with internal dielectric transduction was presented in Weinstein and Bhave (2009). This dielectric transduction was previously shown to be compatible with operational frequencies greater than $5 \mathrm{GHz}$ (Weinstein and Bhave 2009). The internal capacitor is designed by etching a deep-trench within the cavity (e.g. Si cavity) using a similar process to the DRIE-Bosh process. A thin dielectric film is deposited on the side walls (e.g. $\mathrm{Si}_{3} \mathrm{~N}_{4}$ ) to act as the dielectric material of the capacitor. The trench is then filled with a low-loss material having a closely matched acoustic impedance to the bulk (e.g. poly$\mathrm{Si}$ for Si cavities). The manufacturing of such DT capacitors has been developed in Wang and Weinstein (2012a, b) and analytical modeling of the motional characteristics and considerations for the placements of the transducer elements was presented in Weinstein and Bhave (2009).

\section{Fabrication and characterization}

\subsection{Fabrication}

The devices were implemented in a process similar to that previously described for unreleased resonators (Wang and

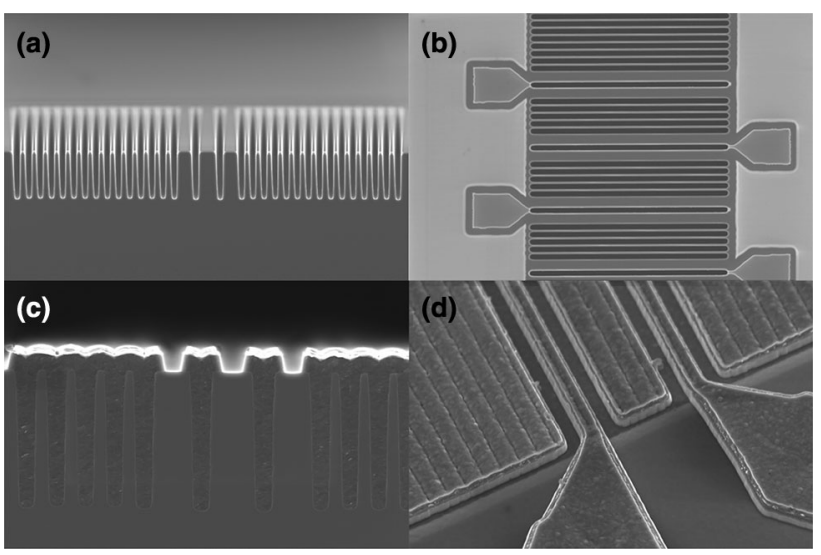

Fig. 13 SEM images of representative devices showing: a etch of the deep trenches in the Si bulk, b PSG deposition and drive-in for the low-resistivity channel, c dielectric $\mathrm{Si}_{3} \mathrm{~N}_{4}$ deposition, poly-Si trench fill and d) field-oxide and metal deposition for the electrical routing

Weinstein 2012b). Figure 13 shows the SEM images of the different process steps for the coupled cavities resonators. Figure $13 \mathrm{~b}$ shows the deposition of phosphorous silicon glass (PSG) which constitutes the n-type doping channel surrounding each of the electrical DT drive/sense capacitors. The process was optimized to obtain a good poly-Si trench fill without voids. These trenches have a significantly larger aspect ratio than those presented in Wang and Weinstein (2012b). Figure 14 shows an optical micrograph of a five cavity resonator fabricated in this process.

\subsection{Process and design recommendations}

To limit the scattering and coupling into spurious modes, one could instead use an inter-digitation of Drive/Sense DT capacitors as used in the metal electrode patterns of SAW resonators, where the pitch is set to $\lambda$ of the acoustic wavelength. If the pitch-size of the transducer DT and ABR are close in length (e.g. within hundreds of $\mathrm{nm}$ ), then there

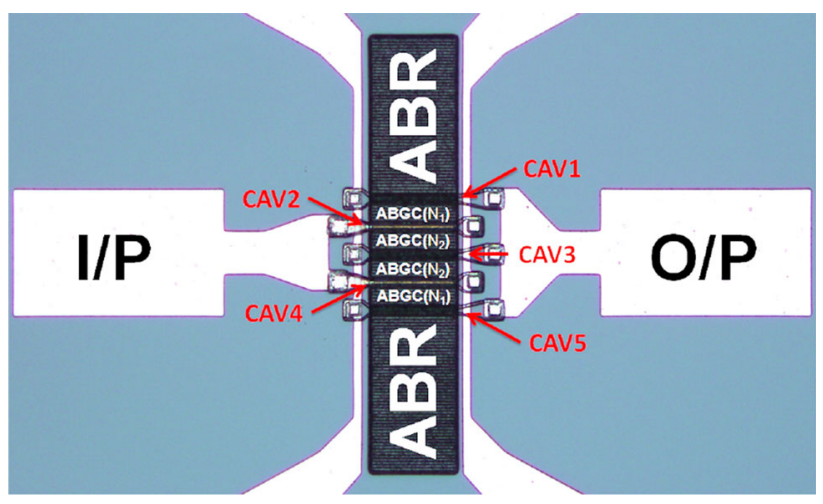

Fig. 14 Optical micrograph of a five coupled cavities design in the $\langle 110\rangle$ direction, with the $\mathrm{ABR}, \operatorname{ABGC}\left(N_{1}\right), \operatorname{ABGC}\left(N_{2}\right)$ and $\mathrm{CAV}$ regions labeled 


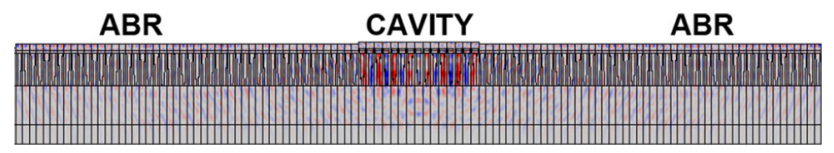

Fig. 15 COMSOL simulated resonant mode shape (strain distribution) at $f_{0}=2.7 \mathrm{GHz}$ model of a single cavity resonator with an interdigitation of drive/sense $\mathrm{dt}$ capacitors

is nearly no structural distinction anymore between the resonant cavities and the reflectors, as it is shown in Fig. 15. The benefits being that the $\mathbf{k}$ wave vector of the driving will be much more localized in the $(k, \omega)$ space, reducing the scattering into other spurious modes existing within the sound cone region. IDT configurations also enable differential driving and sensing schemes which have the potential to significantly reduce the electrical feedthrough.

The numerical optimization of these single cavity resonators, however, showed low simulated $Q$ values, with stringent requirements on having a near perfect-match of the transducer and reflector pitch size. The structures still suffer from the small acoustic mismatch between the $\mathrm{p}-\mathrm{Si}$ and $\mathrm{Si}$, which produces weak acoustic reflectors and allows energy to leak away into the surroundings. Two specific suggestions for process revisions are provided below.

\subsubsection{Silicon dioxide acoustic reflectors}

Instead of using poly-Si filled trenches for the ABR, one solution would be to use $\mathrm{SiO}_{2}$ to boost the acoustic mismatch coefficient to $\frac{Z_{\mathrm{Si}}}{Z_{\mathrm{SiO}_{2}}} \approx 1.75$. However, because of the sharp break in translational symmetry, the simulated $Q<100$. The large band-gap formed by the $\mathrm{SiO}_{2}$ reflectors prevents any energy to leak-away horizontally though the finite ABRs, favoring a direct radiation of the energy by coupling to higher-order modes and scattering it away in the bulk substrate. Other suitable trench re-fill materials could also be investigated to achieve the same purpose.

\subsubsection{ABR reflectors and GRIN structures}

Since a low acoustic impedance material is used (i.e. $\mathrm{SiO}_{2}$ ) in conjunction with poly-Si, there is no need to have a large number of outer ABR reflectors anymore. To diminish the scattering of the waves, gradient-index section (GRIN) can be used between the outer reflectors and the resonant domain to provide for a smooth transition and minimize the break in the translational symmetry. The GRIN section has a gradual increase in the pitch-size from the unit-cell transducer pitch size (e.g. $3.4 \mu \mathrm{m}$ ) to that of the ABR reflectors (e.g. $6.9 \mu \mathrm{m}$ ). The designs have ten GRIN layers with a linear increase in pitch-size. Such gradual structural variations have also been used in Joannopoulos et al. (2008) to improve the $Q$ of the resonators.

\section{Conclusions}

This paper provided the modeling basis and detailed design procedures for the acoustic coupling of unreleased MEMS resonators, employing different transduction schemes. The design of the resonators is based on coupled cavities defined as material regions of integer multiples of acoustic wavelengths in size. The acoustic couplers (ABGC) were produced by periodically stacking together Bragg-unit cells of different sizes and pitch-lengths. These parameters enable a tuning of the coupling strength between adjacent cavities. Coupled resonators using 3, 5 and 7 cavities were fabricated in a CMOS-compatible deep trench micromachining process.

In an attempt to reduce the $R_{x}$ even further, alternative transduction mechanisms using piezoelectric materials can be used. While the structures in this paper have been designed to operate in the $1-3 \mathrm{GHz}$ frequency range, alternative configurations operating at $10 \mathrm{GHz}$ have also been investigated. At such SHF bands, the $R_{x}$ is expected to be significantly lower as more motional current is generated. However, the effect of parasitics at these frequencies on the measured response needs to be investigated as well.

Open Access This article is distributed under the terms of the Creative Commons Attribution 4.0 International License (http://creative commons.org/licenses/by/4.0/), which permits unrestricted use, distribution, and reproduction in any medium, provided you give appropriate credit to the original author(s) and the source, provide a link to the Creative Commons license, and indicate if changes were made.

\section{References}

Auld BA (1990) Acoustic fields and waves in solids, vol 2. R.E. Krieger, Melbourne

Bahr B, Marathe R, Weinstein D (2015) Theory and design of phononic crystals for unreleased CMOS-MEMS resonant body transistors. J Microelectromech Syst 24(5):1520-1533

Erbes A, Thiruvenkatanathan P, Woodhouse J, Seshia AA (2015) Numerical study of the impact of vibration localization on the motional resistance of weakly coupled mems resonators. J Microelectromech Syst 24(4):997-1005

Joannopoulos JD, Johnson SG, Winn JN, Meade RD (2008) Photonic crystals: molding the flow of light, 2nd edn. Princeton University Press, Princeton

Johnson SG, Fan S, Mekis A, Joannopoulos JD (2001) Multipolecancellation mechanism for high-q cavities in the absence of a complete photonic band gap. Appl Phys Lett 78(22):3388-3390

Tamura S, Hurley DC, Wolfe JP (1988) Acoustic-phonon propagation in superlattices. Phys Rev B 38:1427-1449

Thiruvenkatanathan P, Yan J, Woodhouse J, Seshia A (2011) Manipulating vibration energy confinement in electrically 
coupled microelectromechanical resonator arrays. J Microelectromech Syst 20(1):157-164

Wang W, Weinstein D (2011) Acoustic bragg reflectors for Q-enhancement of unreleased MEMS resonators. In: 2011 Joint Conference of the IEEE international frequency control and the European Frequency and Time Forum (FCS), pp 1-6

Wang W, Weinstein D (2012a) Unreleased MEMS resonator and method of forming same, U.S. Patent 20130033 338, 2012

Wang W, Weinstein D (2012b) Deep trench capacitor drive of a 3.3 $\mathrm{GHz}$ unreleased Si MEMS resonator. In: 2012 IEEE international electron devices meeting (IEDM), pp 15.1.1-15.1.4
Weinstein D, Bhave S (2009) Internal dielectric transduction in bulkmode resonators. J Microelectromech Syst 18(6):1401-1408

Publisher's Note Springer Nature remains neutral with regard to jurisdictional claims in published maps and institutional affiliations. 